Münchner Studenten die Grundlagen unserer Rechtsordnung vermittelt. Bisher hat er dort über den Artikel 97 Abs. I des Grundgesetzes nicht doziert. Aber er hat in Berlin bereits klargestellt, wie wichtig ihm die in dieser Verfassungsnom garantierte Unabhängigkeit der Richter ist. In einer öffentlichen Stellungnahme erklärt er, die *Unterstellung * des Vereins der Berliner Verwaltungsrichter, *die Verwaltung habe mit ihrer Personalentscheidung Einfluß auf die Rechtsprechung nehmen wollen $\alpha$, sei sabsurd und unverantwortich*. Eines weiteren Kommentars dazu bedürfe es nicht.

Die Münchner Studenten können stolz sein auf ihren Professor, der es in dieser einzigartigen Weise versteht, wissenschaftlich gründliche und aufrichtige Bemühung um unsere Verfassung mit einer ebensolchen politischen Praxis zu verbinden.

Martin Jacobs

\title{
Zur Sprache der Radikalenentscheidung
}

-Ist auf die Beamtenschaft kein Verlaß mehr, so sind die Gesellschaft und ihr Staat in kritischen Situationen sverloren«*. (BVerfGE 39, S. 349). Vor diesem Hintergrund, der Vorsorge für *kritische Situationen «, sind die Passagen für die Kritik-Möglichkeiten des Beamten zu lesen - es geht, unausgesprochen, zugleich um das Verhältnis von Kritik und Krise. Das Bundesverfassungsgericht ordnet zunächst die politische Treuepflicht* als * Kern * einer Treuepflicht des Beamten überhaupt zu - das ist eine Metapher aus der Natur, sie operiert mit den Bildern von Innen und Außen, vom Weichen, Durchdringlichen und Wandelbaren (Fruchtfleisch etwa kann in der Farbe differieren, vertrocknen, verfaulen, konsumiert werden usw.) und dem Harten, Undurchdringlichen und Unwandelbaren. 'Beamtenkörperc kommen und gehen (seit dem Ende des I 8. Jahrhunderts laut Bundesverfassungsgericht), Staatsformen wechseln wie die Pflanzen, dieser $*$ Kern* aber in der politischen Naturgeschichte der Deutschen bleibt bestehen. Er erfährt im folgenden nähere Bestimmungen, diese:

- Gemeint ist damit nicht eine Verpflichtung, sich mit den Zielen oder einer bestimmten Polizik der jeweiligen Regienung zu identifizieren. Gemeint ist vielmehr die Pflicht zur Bereitschaft, sich mit der Idee des Staates, dem der Beamte dienen soll, mit der freiheitlichen demokrztischen, rechts- und sozialstaatlichen Ordnung dieses Staztes zu identifzieren. Dies schließt nicht aus, an Erscheinungen dieses Staates Kritik üben zu dürfen, für Änderungen der bestehenden Verhältnisse - innerhalb des Rahmens der Verfassung und mit den verfassungsrechtlich vorgesehenen Mitteln - eintreten zu können, solange in diesem Gewand nicht eben dieser Staat und seine verfassungsmäßige Grundlage in Frage gestellt werden. An einer wunkritischen a Beamtenschaft können Staat und Gesellschaft kein Interesse haben. Unverzichtbar ist aber, daß der Beamte den Staat - ungeachtet seiner Mängel - und die geltende verfassungsrechtliche Ordnung, so wie sie in $\mathrm{K}$ raft steht, bejaht, sie als schützenswert anerkennt, in diesem Sinne sich zu ihnen bekennt und aktiv für sie eintritt. Der Beamte, der dies tut, genügt seiner Treuepflicht und kann von diesem Boden aus auch Kritik äußern und Bestrebungen nach Änderungen der bestehenden Verhältnisse - im Rahınen der verfassungsmäßigen Ordnung und auf verfassungsmäßigen Wegen! - unterstützen. * (BVerfGE 39 , S. 347 f.)

Kern der Treuepflicht ist die Identifikation mit einer "Idee des Staates ; die Idee wird freilich in der nachfolgenden Explikation als existierende Ordnung ausgegeben. Es ist nicht zufällig von der Identifizierung mit der „Ordnung dieses Staates* die Rede statt von $\rightarrow$ Achten und Wahren der Verfassunga. Damit ist der - kritische Gedanke, die Idee sei allererst historisch zu realisieren, mit gesellschaftlichem Leben 
zu ertüllen, a lımıne ins Abseıts gestellt und mit ihm auch der Gedanke, zwischen der Idee und der politischen, historisch kontingenten Ordnung könne ein Widerspruch, von Anspruch und Wirklichkeit zum Beispiel bestehen. Nachdem derart ausgeschlossen worden ist, möchte der Text ein Kritikzugeständnis auch für den Beamten formulieren - und kommt in Schwierigkeiten. Denn vom Identifikationsgebot her, sprich: von der behaupteten Identität von Idee und realer Ordnung her läßr sich Kritik an dieser logisch nicht mehr begründeten, noch dazu Kritik aus dem Beamtenkörper, der als Kern die Vermittlung zwischen Idee des Staates und realer Ordnung selber trägt - der Beamtenkörper ist die leibliche Erscheinung der Idee, in ihm legt sie sich, als Idee des Staates eigentlich aller historischen Kontingenz enthoben, in die Realgeschichte als Ordnungs-Geschichte sus.

Kritik soll aber sein dürfen, denn dunkel mag auch dem Gericht noch bexußt sein, daß Kritik und Kritikfähigkeit konstitutiv zur Idee jener Staatsform gehört, in deren zeitliches Gewand - nach Monarchie und konstiturioneller Monarchie (eine r zjährige deutsche Staatsform im 20. Jahrhundert findet das Bundesverfassungsgericht nicht erwähnenswert, wahrscheinlich weil zu dieser Zeit die Treuepflicht des Beamten gänzlich unproblematisch war. Vgl. auch Hegel: die Perioden des Glücks sind leere Blätter in der Geschichte) - die Idee des Staates sich gerade gehüllt hat: der demokratischen Republik nämlich. Da es im Grunde schon ausgeschlossen hat, fährt es - seine Sprache selber übertührt es - folgerichtig fort: „Dies schließt nicht aus, an Erscheinungen dieses Staates Kritik üben zu dürfen * - wie aber, wenn es das Wesen wäre, was da erschiene? Und das Wesen muß erscheinen (ist nicht im Satz zuvor die Identitär von Wesen - Idee des Staates - und Erscheinung - realer staatlicher Ordnung - behaupter worden, und das entspräche ja auch der Legitimationslogik des Gerichts?). Aber es fährr, kühn geworden, noch ein Stück fort, es wagt sich an eine inhaltliche Bestimmung von Kritik: „für Änderungen der bestehenden Verhältnisse* - an dieser Stelle aber findet der Aufschwung des kritischen Gedankens ein jähes Ende, er stürz förmlich in den Rahmen der realexistierenden Idee, die gebieterisch ihre Präsenz einklagt: - innerhalb des Rahmens der Verfassung und mit den verfassungsrechtlich vorgesehenen Mitteln - , erst nach der Parenthese kommt er zur Ruhe: »eintreten zu können*. Es steht aber kein Punkt da, sondern ein », a und danach das Wort: *solange* - eine zeitliche Bestimmung? wäre es denkbar? nun würde historisch gedacht - solange bis (sagen wir mal versuchsweise) die Ideen der Verfassung reale gesellschaftliche Gestalt angenommen hätten?

Aber so ist es nicht gemeint, das solange* hat eine ganz andere Funktion, eine konditionale, es stellt die Kritik, das Eintreten für Änderungen der bestehenden Verhältnisse, unter einen prinzipiellen Vorbehalt, unter eine Duldungsklausel und lieferr sie gänzlich dem Interpretationsmonopol des Verfassungsrechts aus: »solange in diesem Gewand nicht eben dieser Staat und seine verfassungsmäßige Grundlage in Frage gestellt werden.* Und nun erst steht da ein Punkt.

Wie also? Der eben auf Erscheinungen als ihren Gegenstand verpflichteten Kritik (schwer, da nicht an Karl Valentin zu denken, der, befragt, warum er eine Brille mit Fensterglas trüge, antwortete: $\bullet$ Besser ist es doch als gar nichts.«), ihr wird bedeutet, ihr - immerhin benannter - konkreter Inhalt stehe unter dem prinzipiellen Verdacht, selber nur Schein, Verstellung, Verhüllung zu sein. Wovon? Von einer Absicht, einer Intention, die aber doch zugleich konstitutiv für sie ist, ohne die sie nicht wäre und ihren Namen nicht verdiente, nämlich der: in Frage zu stellen, und zwar gerade auch Ideen, die sich als mit politisch-gesellschaftlichen Ordnungen identisch vorstellen, oder anders: diesen Staat und seine verfassungsmäßige Grundlage, insofern jener als politische Ordnung mit dieser als Idee der Demokratie noch keineswegs konkret gesellschaftlich identisch ist. 
Noch eben zugestanden wird die Kritik so an cine Schranke geführt, mehr: es wird ihr eine Schranke imputiert, die Schranke des Gerichts nämlich, die mitzuschleifen ihre Möglichkeiten auf eine kümmerliche Stufe, eine Schwundstufe gewissermaßen, reduziert. Was folgt ist der kürzeste Satz im Urteilstext überhaupt - und er hat so etwas fatal Flaues, so wenig Begeistertes und Begeisterndes und auch so wenig Geist, er schleppt sich selber schwindsüchtig dahin -: $\gg$ An einer unkritischen Beamten. schaft können Staat und Gesellschaft kein Interesse haben.* Er sagt nicht: An einer kritischen Beamtenschaft müssen Staat und Gesellschaft in einer Demokratie ein lebensnotwendiges Interesse haben, weil Demokratie als Idee auf einen Prozeß, auf mündige politische Betciligung aller hin angelegt ist, nein, das mag er wohl auch nicht sagen - wegen des Gewandes namlich -.

Wie hymnisch, syntaktisch prunkvoll und reich an Bestimmungen wird, nach diesem Schwächeanfall, die juristische Sprache nun, sie ist wieder auf ihrem ureigenen Feid, dem Dekretieren von positivem Verhalten. Und im Schwung der Positivität kassiert sie nun gänzlich, was eben noch dürftig genug zugestanden war: die Kritik.

Diese Sprache, die von der Möglichkeit der Kritik allenfalls in doppelten Negationen und mit Kann-Bestimmungen reden mag, sie überschlägt sich förmlich, wenn es um die Affirmation der bestehenden Ordnung und den Staat geht, vier Prädikate bieter sie auf: bejahen, anerkennen, bekennen, aktiv eintreten. Über die empirischen Niederungen seiner Mängel hinweg hebt sie den Beamten in den Wertehimmel des Staates - und er bejaht, er erkennt an, er bekennt, ja er tritt aktiv ein. $*$ - Ungeachtet seiner Mängel - will sagen: er soll sciner Mängel nicht achten, nicht eigenbrötlerisch am Negativen kleben, das es hie und da noch geben mag, es geht ums Ganze und das Ganze ist der Staat. Und die Kritik? - eh schon dahinsiechend an ihrer imputierren Schranke, nun liegt sie gänzlich am Boden: Und das ist zugleich der $\gg$ Boden $*$, von dem aus sie »auch « geäußert werden $*$ kann .

Solche juristische Syntax hat eine Physiognomie, ihr Äußeres ist auf ein Inneres hin lesbar geworden. Das innere Bild aber, das sich in diesen Sätzen ausprägt, ist das einer Festung - der Konditionalis ist ihr Glacis, die Parenthesen sind ihre Laufgräben, die abwandelnden Wiederholungen ihre Schießscharten und das Ausrufezeichen ihre Fahne.

Nicht genug damit, daß Kritik - die intellektuelle Fähigkeit zur Differenzerfahrung - so auf eine Schwundstufe gebracht ist, im folgenden geht das Bundesverfassungsgericht dann zur festlegenden Beschreibung innerer, emotionaler Positivität über (»mehr als nur eine formal korrekte, im übrigen uninteressierte, kühle, innerlich distanzierte Haltung gegenüber Staat und Verfassung *), leiret daraus das Distanzierungsgebot gegenüber kritischen Bestrebungen ab und schließt wiederum mit einer Metapher, dem Bild des dienenden Beamten, der sich im Staate, in seinem Staate $* z u$ Hause fühlt - jetzt und jederzeit und nicht erst, wenn die von ihm erstrebten Veränderungen durch entsprechende Verfassungsänderungen verwirklicht worden sind. « (BVerfGE 39, S. 348) Damit soll - ergänzend zur Kritik - auch die emotionale Fähigkeit zur Differenzerfahrung, z. B. die Spontaneität eines verletzten Rechtsbewußtseins, Zorn, Betroffenheit auf Dauer stillgestellt werden.

Heinz Brüggemann 